\title{
Presence of selected metabolic syndrome components in patients with psoriasis vulgaris
}

\author{
Sebastian Uczniak, Zofia A. Gerlicz, Magdalena Kozłowska, Andrzej Kaszuba
}

Department of Dermatology, Pediatric Dermatology and Oncology, Medical University of Lodz, Lodz, Poland

Adv Dermatol Allergol 2016; XXXIII (2): 114-119

DOI: 10.5114/ada.2016.59153

\begin{abstract}
Introduction: Recent studies have suggested a strong association between psoriasis and obesity, dyslipidemia, hypertension, resistance to insulin and metabolic syndrome.

Aim: To assess the prevalence of selected metabolic syndrome components in patients with psoriasis and the effect of the abnormalities on the disease activity.

Material and methods: Two hundred and forty-six patients diagnosed with psoriasis and 75 healthy individuals as controls were included in the study. Psoriasis activity was evaluated by the Psoriasis Area and Severity Index (PASI). Results: There was a statistically significant difference in triglyceride concentration between psoriasis patients and controls ( $p=0.00001)$, which was not found for high-density lipoprotein (HDL) concentration. Mean values of serum glucose level in patients with psoriasis were significantly higher than in controls $(p=0.046)$. Further statistical analysis of the obtained results showed significantly higher systolic blood pressure in the psoriasis patients than in the controls $(p=0.0001)$, but there was no statistically significant difference in diastolic blood pressure between the investigated groups $(p>0.05)$.

Conclusions: Higher prevalence of metabolic syndrome components was observed in patients with psoriasis than in the general population.
\end{abstract}

Key words: psoriasis, metabolic syndrome, dyslipidemia, hypertension.

\section{Introduction}

Metabolic syndrome was defined for the first time by Gerald Reaven in 1988 [1]. It is the name for co-occurrence of interrelated metabolic disorders raising the risk of developing cardiovascular diseases and diabetes type 2 . The beginning of the term dates back to the attempts to isolate a group of risk from a seemingly healthy population [1, 2]. Despite its long history of over 25 years, the diagnosis of metabolic syndrome is still not clear, and diagnostic criteria do not directly reflect clinical changes but are only an arbitrary attempt to establish norms. All markers designating metabolic syndrome (Table 1), irrespective of the adopted criteria, are characterized by increasing the risk of cardiovascular disease. It has been estimated that in the Polish population metabolic syndrome is present in about $20 \%$ of the investigated individuals $[3,4]$. Therefore proper diagnosis of metabolic syndrome may enable prevention and treatment of complications, thus contributing to a decrease in development of metabolic and circulatory diseases.

Psoriasis is a common chronic inflammatory disease associated with serious comorbidities. In the past it used to be defined as chronic relapsing inflammatory skin disease with mildly increased proliferation of epidermal keratinocytes. At present psoriasis is known to affect not only skin, but from $5 \%$ up to $30 \%$ of patients develop psoriatic arthritis, and other internal organs may also be affected [2]. Clinical observation and scientific research have found that metabolic syndrome is more common in psoriatic patients [5-8]. Increase in the risk of developing hypertension, diabetes and hyperlipidemia is statistically significant. Abdominal obesity is also more common in these patients [9]. Moreover, disorders found in patients with metabolic syndrome such as insulin resistance, hyperinsulinemia, endothelial dysfunction, chronic inflammation, increase in oxidative stress, abdominal obesity, dysregulated adipokine secretion and non-alcoholic fatty 
liver disease are also observed in psoriatic patients. Psoriasis and cardiovascular diseases seem to have common etiopathogenetic features and may be mediated by the same proinflammatory cytokines (e.g., TNF- $\alpha, \mathrm{IL}-1$, IL-6, IL-8, IL-12). It raises the question whether metabolic syndrome contributes to psoriasis manifestation or on the contrary development of psoriasis promotes metabolic disorders and increases the risk of cardiovascular diseases.

\section{Aim}

The aim of the study was to determine prevalence of the selected metabolic syndrome markers and their relationship with psoriasis severity.

\section{Material and methods}

\section{Study participants}

One hundred and eight females (F) and 138 males (M) with chronic plaque psoriasis and 75 (40 F and $35 \mathrm{M}$ ) healthy controls were recruited from the Department of Dermatology, Pediatric Dermatology and Oncology of Medical University in Lodz. The mean age was $46 \pm 13$ years. The medical history and clinical examination were done. Healthy controls did not have psoriasis or any systemic diseases.

\section{Anthropometric and laboratory measurements}

Waist circumference of the participants was measured from the upper margin of the posterior iliac crest at the end of normal expiration directly above the skin. Weight and height were recorded. Brachial blood pressure was measured. The clinical severity of psoriasis was assessed using the Psoriasis Area and Severity Index (PASI), on the first visit. Blood samples were collected after overnight fasting. Lipid profile and fasting blood glucose (FBG) were measured by an automated clinical chemistry analyzer.

The study was approved by the Ethical Committee of the Medical University of Lodz.

\section{Statistical analysis}

Statistical significance was analyzed by the non-parametric Mann-Whitney test, Student's t-test and Spearman's rank correlation test. $P$-values less than 0.05 were considered significant.

\section{Results}

There was a statistically significant difference in triglyceride concentration between psoriasis patients and the controls ( $p=0.00001$ ), which was not found for HDL concentration (Table 2). Mean values of serum glucose level in patients with psoriasis were significantly higher than in the controls $(p=0.046)$. Further statistical analysis of the obtained results showed significantly higher systolic blood pressure in the psoriasis patients than in the controls ( $p=0.0001$ ), but there was no statistically significant difference of diastolic blood pressure between the investigated groups ( $p>0.05$ ) (Table 2).

Mean values of waist circumference of males were significantly higher in psoriasis patients than in controls $(p=0.0023)$. A similar relationship was found for waistto-hip ratio (WHR) ( $p=0.049)$ (Table 2$)$.

The results obtained in the investigations carried out on patients with psoriasis showed a relationship between increased serum glucose level and severity of psoriatic lesions $>20$ in the PASI score. Males with severe psoriasis $>20$ in the PASI score showed significantly higher serum HDL level and triglyceride (TG) than in males with milder skin lesions $<20$ PASI ( $p=0.02$ and $p=0.02$, respectively). There was a statistically significant difference in serum HDL level between males and females with psoriasis and skin lesions $<20$ in the PASI score. There was also a difference in WHR between females and males in both groups, PASI $<20$ and PASI $>20 ; p=0.002$ and $p=0.009$, respectively.

Further statistical analysis showed a significant relationship between values of systolic blood pressure in males with severe psoriasis PASI > 20 and those with PASI $<20(p=0.032)$ and also a difference between values of diastolic blood pressure in females with severe psoriasis PASI $>20$ and those with PASI $<20(p=0.004)$. There was also a statistically significant difference in systolic blood pressure values in psoriasis females and males with PASI > 20 and values of diastolic blood pressure between female and male patients with PASI $<20$ and PASI > 20 (Table 3 ).

Table 1. Metabolic syndrome - Polish Forum for Prevention of Cardiovascular Diseases criteria (2009)

\begin{tabular}{lcc}
\hline \multicolumn{2}{l}{ Waist circumference plus $\geq 2$ risk factors: } \\
\hline $\begin{array}{l}\text { Abdominal obesity: } \\
\text { Waist circumference }\end{array}$ & Male $(M)$ & $\geq 94 \mathrm{~cm}$ \\
\cline { 2 - 3 } & Female $(\mathrm{F})$ & $\geq 80 \mathrm{~cm}$ \\
\hline Risk factor: & Fasting glucose \\
\hline Insulin resistance & $\geq 100 \mathrm{mg} / \mathrm{dl}$ \\
\hline $\begin{array}{l}\text { Dyslipidemia or } \\
\text { hyperlipidemia } \\
\text { treatment }\end{array}$ & Triglycerides & $\geq 150 \mathrm{mg} / \mathrm{dl}$ \\
\cline { 2 - 3 } & Cholesterol HDL (M) & $<50 \mathrm{mg} / \mathrm{dl}$ \\
\cline { 2 - 3 } & Cholesterol HDL (F) & $<40 \mathrm{mg} / \mathrm{dl}$ \\
\hline $\begin{array}{l}\text { Hypertension } \\
\text { or hypotension } \\
\text { treatment }\end{array}$ & \begin{tabular}{c} 
Systolic blood pressure \\
\cline { 2 - 3 }
\end{tabular} & $\begin{array}{c}\text { Diastolic blood } \\
\text { pressure }\end{array}$ \\
\hline
\end{tabular}


Table 2. Assessment of metabolic syndrome parameters in psoriasis patients and controls

\begin{tabular}{|c|c|c|c|c|c|c|c|c|c|c|c|}
\hline \multirow[t]{2}{*}{ Parameter } & \multicolumn{5}{|c|}{ Psoriasis } & \multicolumn{5}{|c|}{ Control group } & \multirow[t]{2}{*}{$P$-value } \\
\hline & $N$ & $\bar{x}$ & SD & Min. & Max. & $N$ & $\bar{x}$ & SD & Min. & Max. & \\
\hline TG & 246 & 145.68 & 29.95 & 77 & 210 & 75 & 110.92 & 28.83 & 35 & 155 & $<0.00001$ \\
\hline $\mathrm{HDL}$ & 246 & 58.74 & 14.56 & 38 & 116 & 75 & 58.17 & 13.49 & 43 & 104 & * \\
\hline Fasting glucose & 246 & 96.71 & 16.02 & 66 & 135 & 75 & 92.64 & 13.17 & 66 & 115 & 0.046 \\
\hline Waist circum.: & 246 & 97.03 & 13.71 & 65 & 138 & 75 & 92.00 & 14.42 & 62 & 120 & 0.006 \\
\hline Female & 108 & 95.00 & 14.14 & 65 & 126 & 45 & 91.2 & 16.35 & 62 & 120 & * \\
\hline Male & 138 & 99.04 & 1.87 & 72 & 138 & 30 & 93.2 & 11.67 & 70 & 120 & 0.023 \\
\hline WHR: & 246 & 0.9 & 0.12 & 69.00 & 1.19 & 75 & 0.87 & 0.08 & 0.73 & 1.04 & 0.043 \\
\hline Female & 108 & 0.87 & 0.1 & 0.69 & 1.1 & 45 & 0.87 & 0.069 & 0.75 & 0.97 & * \\
\hline Male & 138 & 0.93 & 0.13 & 0.7 & 1.19 & 30 & 0.88 & 0.1 & 0.73 & 1.04 & 0.049 \\
\hline Systolic BP & 246 & 135.06 & 12.82 & 115 & 165 & 75 & 128.8 & 9.6 & 110 & 140 & 0.0001 \\
\hline Diastolic BP & 246 & 83.26 & 6.16 & 70 & 90 & 75 & 83.00 & 6.92 & 70 & 90 & * \\
\hline
\end{tabular}

$T G$ - triglycerides, HDL - high-density lipoprotein, Waist circum. - waist circumference, WHR - waist to hip ratio, BP-blood pressure, SD - standard deviation. *If not specified: $p>0.05$.

\section{Discussion}

There is a substantial number of studies showing that patients with psoriasis have an increased incidence of metabolic syndrome including abdominal obesity, insulin resistance, decreased HDL cholesterol, hypertriglyceridemia, and hypertension [3, 6].

Obesity is a known risk factor for psoriasis development and might be correlated with psoriasis activity. Psoriasis patients have higher prevalence and incidence of obesity, and those with more severe skin lesions have higher odds of obesity compared to those with mild psoriasis [10, 11]. Interestingly, Wolk et al. [12] established that for each unit increment increase in body mass index (BMI), there was a 9\% higher risk for psoriasis onset and $7 \%$ higher risk for PASI. Recent studies show that WHR has a stronger correlation with cardiovascular diseases [13]. Our study also showed associations between psoriasis and obesity $(p=0.43)$ with high WHR compared with the general population. Moreover, we observed that most men remained in the group at high risk of developing visceral obesity. Our results are in accordance with the cross-sectional study by Huang et al. [14] in which a linear trend for increasing psoriasis severity across increasing body mass index (BMI) groups was observed and was greater in men than women. The obtained data suggest that excess adiposity may be a risk factor of psoriasis as well as metabolic syndrome and cardiovascular morbidity risk. Moreover, a recent study by Li et al. found that the association between polymorphism in IL-12B and psoriasis risk may be modified by measures of overall and central adiposity [15].

Abdominal obesity may coexist with atherogenic hyperlipidemia. Interestingly, the pattern of dyslipidemia found in metabolic syndrome, which features elevated triglycerides and low HDL cholesterol, has been reported in association with psoriasis and psoriatic arthritis [16]. In the study we observed that hypertriglyceridemia was associated with an elevated risk of severe psoriasis (PASI $>20)$ but only in male groups $(p<0.5)$. The observed strong association between hypertriglyceridemia and psoriasis in comparison with the control group suggests an effect of dyslipidemia on psoriasis. Conversely, pro-inflammatory activities observed in psoriasis may influence abnormalities of plasma lipid levels. In the El Asmi et al. analysis [17] of a Tunisian psoriatic group, serum levels of TG, low-density lipoprotein (LDL) and very low density lipoprotein (VLDL) cholesterol were also significantly higher than in the control group $(p<0.05)$, while the HDL was significantly decreased in patients with psoriasis compared to the controls $(p<0.001)$. Similarly, hypertriglyceridemia and abdominal obesity $(p<0.05)$ were strongly associated with psoriasis in the Damevska et al. study [18]. The same findings were observed in a systematic review of 25 cross-sectional and case-control studies: psoriasis was associated with greater odds of dyslipidemia [19]. Further, He et al. [20] showed that biological activities of psoriatic lipoproteins are impaired in both HDL and LDL and hypothesized that it may provide a link between psoriasis and cardiovascular diseases.

Hypertension is more often observed in patients with psoriasis compared to the control population [21]. Conversely, long-term hypertensive status is associated with an increased risk of psoriasis [22], although this association remains controversial. In our study, patients with severe psoriasis are at a higher risk of hypertension than those with mild psoriasis $(p<0.05)$. Interestingly, 
Table 3. Assessment of metabolic syndrome parameters depending on severity of psoriatic lesions

\begin{tabular}{|c|c|c|c|c|c|c|c|c|c|c|c|}
\hline \multirow[t]{2}{*}{ Parameter } & \multicolumn{5}{|c|}{ PASI $<20$} & \multicolumn{5}{|c|}{ PASI $>20$} & \multirow[t]{2}{*}{$P$-value } \\
\hline & $N$ & $\bar{x}$ & SD & Min. & Max. & $N$ & $\bar{x}$ & SD & Min. & Max. & \\
\hline TG: & 147 & 147.38 & 28.11 & 92 & 210 & 99 & 151.37 & 27.01 & 101 & 200 & * \\
\hline Female & 66 & 148.31 & 26.37 & 98 & 210 & 42 & 144.78 & 31.16 & 102 & 196 & * \\
\hline Male & 81 & 147.07 & 30.39 & 92 & 200 & 57 & 164.15 & 25.83 & 101 & 200 & 0.0007 \\
\hline \multicolumn{5}{|c|}{ * } & \multicolumn{7}{|c|}{ Fvs. $M p=0.001$} \\
\hline HDL: & 147 & 60.45 & 14.91 & 39 & 116 & 99 & 57.01 & 13.62 & 38 & 101 & * \\
\hline Female & 66 & 51.59 & 14.18 & 39 & 79 & 42 & 56.15 & 11.46 & 38 & 84 & * \\
\hline Male & 81 & 64.18 & 15.15 & 42 & 116 & 57 & 57.89 & 15.92 & 46 & 101 & 0.020 \\
\hline \multicolumn{6}{|c|}{ Fvs. $M p=0.0001$} & * & & & & & \\
\hline Fasting glucose: & 147 & 94.09 & 12.88 & 65 & 135 & 99 & 100.08 & 17.45 & 72 & 174 & 0.002 \\
\hline Female & 66 & 95.95 & 11.28 & 75 & 120 & 42 & 102.28 & 25.41 & 88 & 174 & * \\
\hline \multirow[t]{2}{*}{ Male } & 81 & 93.92 & 14.63 & 65 & 135 & 57 & 97.47 & 13.95 & 72 & 132 & * \\
\hline & * & & & & & * & & & & & \\
\hline Waist circum.: & 147 & 95.99 & 13.81 & 62 & 138 & 99 & 97.21 & 13.77 & 65 & 126 & * \\
\hline Female & 66 & 97.55 & 14.90 & 72 & 138 & 42 & 95.78 & 16.56 & 65 & 126 & * \\
\hline \multirow[t]{2}{*}{ Male } & 81 & 95.99 & 13.81 & 62 & 138 & 57 & 100.1 & 10.85 & 84 & 123 & * \\
\hline & * & & & & & * & & & & & \\
\hline WHR: & 147 & 0.89 & 0.117 & 0.69 & 1.13 & 99 & 0.91 & 0.121 & 0.77 & 1.24 & * \\
\hline Female & 66 & 0.86 & 0.10 & 0.69 & 1.1 & 42 & 0.88 & 0.08 & 0.77 & 1.06 & * \\
\hline \multirow[t]{2}{*}{ Male } & 81 & 0.92 & 0.13 & 0.70 & 1.13 & 57 & 0.94 & 0.13 & 0.79 & 1.24 & * \\
\hline & & \multicolumn{3}{|c|}{ Fvs. $M p=0.002$} & \multicolumn{6}{|c|}{ Fvs. $M p=0.009$} & \\
\hline Systolic BP: & 147 & 134.91 & 11.89 & 110 & 160 & 99 & 135.11 & 13.54 & 110 & 165 & * \\
\hline Female & 66 & 136.81 & 12.30 & 110 & 160 & 42 & 132.85 & 13.25 & 110 & 160 & \\
\hline \multirow[t]{2}{*}{ Male } & 81 & 133.70 & 11.73 & 115 & 160 & 57 & 138.42 & 13.74 & 115 & 165 & 0.032 \\
\hline & * & & & & \multicolumn{7}{|c|}{ Fvs. $M p=0.046$} \\
\hline Diastolic BP: & 147 & 84.12 & 6.11 & 70 & 90 & 99 & 83.07 & 7.12 & 70 & 100 & * \\
\hline Female & 66 & 85.45 & 6.15 & 75 & 90 & 42 & 81.07 & 8.36 & 70 & 90 & 0.004 \\
\hline \multirow[t]{2}{*}{ Male } & 81 & 83.33 & 6.04 & 70 & 90 & 57 & 84.21 & 5.59 & 70 & 90 & * \\
\hline & & \multicolumn{3}{|c|}{ Fvs. $M p=0.037$} & \multicolumn{7}{|c|}{ Fvs. $M p=0.027$} \\
\hline
\end{tabular}

TG - triglyceride, HDL - high-density lipoprotein, Waist circum. - waist circumference, WHR - waist-to-height-ratio, BP-blood pressure, SD - standard deviation. ${ }^{\star}$ If not specified: $p>0.05$.

we found that men and women with psoriasis do not seem to have equal risk of developing hypertension. Men are more prone to have elevated systolic blood pressure, whereas women are more prone to have elevated diastolic blood pressure. The results of our study support the previously noted association between psoriasis and hypertension.

Presence of glycemic abnormalities in metabolic syndrome, and whether visceral obesity and insulin resistance are essential components of the disorder, still remain controversial [23, 24]. However, obesity is a major risk factor of developing diabetes mellitus type 2 , and a chronic high level of inflammatory adipocytokines is also considered to play a role in stimulating psoriasis activity [25]. On the other hand, psoriasis is thought to promote insulin resistance by overproduction of Th1 cytokines [26]. The relationship between psoriasis and insulin resistance has also been well documented. In an observation study of nearly 65,500 patients, Brauchli et al. [27] found that the risk of incident diabetes mel- 
litus was higher in patients with psoriasis than in the psoriasis-free group. The risk increased with psoriasis duration and severity and, importantly, was not driven by high BMI alone. However, our analysis did not find association between high glucose level and severity of psoriasis at the time of examination, which may be due to short-term observation. A large difference of fasting glucose level was noted between patients with psoriasis and the control group $(p<0.5)$. Indeed, several studies have consistently underlined a significant link between impaired glucose metabolism and psoriasis [23].

Our study also showed a gender difference of metabolic abnormalities within the psoriasis group. Systolic and diastolic pressure differences in psoriasis gender groups are prominent $(p<0.05)$. A similar correlation was observed to be significant for dyslipidemia. Interestingly, also Zindancỳ et al. [5] and Mebazaa et al. [28] found increased prevalence of metabolic syndrome in female patients. Lakshmi et al. [29] observed higher mean BMI and waist circumference in females than in males, but the difference was not statistically significant $(p>0.05)$. Our findings show statistically significant higher waist circumference in males than females $(p<0.05)$, confirming that abdominal obesity is observed more commonly in men, whereas women typically accumulate fat around the hips and thighs. Regardless of the method of measuring obesity, high waist circumference and WHR are predictors of higher risk of cardiovascular disease and psoriasis severity.

\section{Conclusions}

Our findings suggest that psoriasis patients have increased prevalence of the core components of metabolic syndrome. Thus identification of biomarkers related to comorbidities in psoriasis, as well as metabolic syndrome, should be of special clinical interest. Hence, a dermatologist treating psoriasis should be aware of the possible association between metabolic disorders such as hypertension, dyslipidemia or insulin resistance and psoriasis.

\section{Acknowledgments}

This study was supported by Medical University of Lodz grant no. 5-064-01-502-54-039.

\section{Conflict of interest}

The authors declare no conflict of interest.

\section{References}

1. Reaven GM. Banting lecture 1988. Role of insulin resistance in human disease. Diabetes 1988; 37: 1595-607.
2. Pastuszka M, Tyc-Zdrojewska E, Uczniak S, et al. Current concepts of aetiopathogenesis of psoriasis. Postep Derm Alergol 2011; Suppl. 3: 117-31.

3. Broda G, Szczęśniewska D, Rywik S. Częstość występowania zespołu metabolicznego w populacji osób dorosłych Warszawy. Med Metab 2003; 2: 25-9.

4. Zdrojewski T, Bandosz P, Szpakowski P, et al. Rozpowszechnienie głównych czynników ryzyka chorób układu sercowo-naczyniowego w Polsce. Wyniki badania NATPOL PLUS. Kardiol Pol 2004; 61: 5-26.

5. Zindancý I, Albayrak O, Kavala M, et al. Prevalence of metabolic syndrome in patients with psoriasis. Sci World J 2012; 2012: 312463.

6. Kim GW, Park HJ, Kim HS, et al. Analysis of cardiovascular risk factors and metabolic syndrome in Korean patients with psoriasis. Ann Dermatol 2012; 24: 11-5.

7. Owczarczyk-Saczonek AB, Nowicki R. The association between smoking and the prevalence of metabolic syndrome and its components in patients with psoriasis aged 30 to 49 years. Postep Derm Alergol 2015; 32: 331-6.

8. Owczarczyk-Saczonek AB, Nowicki R. Prevalence of cardiovascular disease risk factors, and metabolic syndrome and its components in patients with psoriasis aged 30 to 49 years. Postep Derm Alergol 2015; 32: 290-5.

9. Santos M, Fonseca HM, Jalkh AP, et al. Obesity and dyslipidemia in patients with psoriasis treated at a dermatologic clinic in Manaus. An Bras Dermatol 2013; 88: 913-6.

10. Ni C, Chiu MW. Psoriasis and comorbidities: links and risks. Clin Cosmet Investig Dermatol 2014; 17: 119-32.

11. Armstrong AW, Harskamp CT, Armstrong EJ. The association between psoriasis and obesity: a systematic review and meta-analysis of observational studies. Nutr Diabetes 2012; 2: e54.

12. Wolk K, Mallbris L, Larsson P, et al. Excessive body weight and smoking associates with a high risk of onset of plaque psoriasis. Acta Derm Venereol 2009; 89: 492-7.

13. Duarte GV, Silva LP. Correlation between psoriasis' severity and waist-to-height ratio. An Bras Dermatol 2014; 89: 846-7.

14. Huang YH, Yang LC, Hui RY, et al. Relationships between obesity and the clinical severity of psoriasis in Taiwan. J Eur Acad Dermatol Venereol 2010; 24: 1035-9.

15. Li WQ, Han JL, Zhang MF, Qureshi AA. Interactions between adiposity and genetic polymorphisms on the risk of psoriasis. Br J Dermatol 2013; 168: 639-42.

16. Wu S, Li WQ, Han J, et al. Hypercholesterolemia and risk of incident psoriasis and psoriatic arthritis in US women. Arthritis Rheumatol 2014; 66: 304-10.

17. El Asmi MA, Zidi W, Mebazaa A, et al. Serum lipid level in Tunisian patients with psoriasis. Clin Lab 2014; 60: 1043-7.

18. Damevska K, Neloska L, Gocev G, et al. Metabolic syndrome in untreated patients with psoriasis: case-control study. J Dtsch Dermatol Ges 2013; 11: 1169-75.

19. Ma C, Harskamp CT, Armstrong EJ, et al. The association between psoriasis and dyslipidaemia: a systematic review. Br J Dermatol 2013; 168: 486-95.

20. He L, Qin S, Dang L, et al. Psoriasis decreases the anti-oxidation and anti-inflammation properties of high-density lipoprotein. Biochim Biophys Acta 2014; 1841: 1709-15.

21. Armstrong AW, Harskamp CT, Armstrong EJ. The association between psoriasis and hypertension: a systematic review and meta-analysis of observational studies. J Hypertens 2013; 31: 433-42.

22. Gottlieb AB, Dann F. Comorbidities in patients with psoriasis. Am J Med 2009; 122: 1150.e1-9. 
23. Brauchli YB, Jick SS, Meier CR. Psoriasis and the risk of incident diabetes mellitus: a population-based study. Br J Dermatol 2008; 159: 1331-7.

24. Li W, Han J, Hu FB, et al. Psoriasis and risk of type 2 diabetes among women and men in the United States: a populationbased cohort study. J Invest Dermatol 2012; 132: 291-8.

25. Azfar RS, Seminara NM, Shin DB, et al. Increased risk of diabetes mellitus and likelihood of receiving diabetes mellitus treatment in patients with psoriasis. Arch Dermatol 2012; 148: 995-1000.

26. Onumah N, Kircik LH. Psoriasis and its comorbidities. J Drugs Dermatol 2012; 11: s5-10.

27. Brauchli YB, Jick SS, Meier CR. Psoriasis and the risk of incident diabetes mellitus: a population-based study. Br J Dermatol 2008; 159: 1331-7.

28. Mebazaa A, El Asmi M, Zidi W, et al. Metabolic syndrome in Tunisian psoriatic patients: prevalence and determinants. J Eur Acad Dermatol Venereol 2011; 25: 705-9.

29. Lakshmi S, Nath AK, Udayashankar C. Metabolic syndrome in patients with psoriasis: a comparative study. Indian Dermatol Online J 2014; 5: 132-7. 